\title{
Effects of a 6-week core stabilization training program on dynamic balance and trunk stabilization endurance of horse riders
}

\author{
Nicholas H. K. Lam ${ }^{1}$ (D) Wai Man $\operatorname{Lau}^{2} \cdot$ Tin Lap Lau ${ }^{2}$
}

Received: 7 May 2020 / Accepted: 26 December 2020 / Published online: 20 January 2021

(c) The Author(s) 2021

\begin{abstract}
The purpose of this study was to examine the effectiveness of a 6-week core stabilization training program (CSTP) in improving dynamic balance and back-extensor endurance of horse riders; and develop a measurement tool in assessing the dynamic postural stabilization endurance for horse riders. Twelve male horse riders (age: $23.58 \pm 1.93$ years; height: $165.09 \pm 4.60 \mathrm{~cm}$; weight: $56.53 \pm 4.75 \mathrm{~kg}$; experience in horse racing: $1.75 \pm 0.34$ years) completed 12 training sessions in 6 weeks. Subjects performed the CSTP with two progressions. CSTP started with the basic consciousness activation exercises, and then eliminated visual feedback in dynamic balance task on unstable surfaces and finished with switching the center of gravity. Moderate-to-large difference was demonstrated in the Y-Balance test scores for right leg $(102.81 \pm 8.32$ vs $106.471 \pm 4.35 \mathrm{~cm}, d=0.55,95 \%$ CL 0.00 to 1.08$)$ and left leg $(102.04 \pm 3.20$ vs $106.29 \pm 3.62 \mathrm{~cm}, d=1.25,95 \%$ CL 0.41 to 2.05 ) following 6 weeks CSTP. However, trivial to small differences was reported between left and right leg in pre $(d=0.12,95 \% \mathrm{CL}-0.49$ to 0.73$)$ and post 6-week CSTP $(d=0.04,95 \% \mathrm{CL}-0.69$ to 0.78$)$. Biering-Sørensen test (BST) shows largely greater performance after 6-week CSTP (98.3 \pm 30.1 vs $131.8 \pm 19.0 \mathrm{~s}, d=1.33,95 \%$ CL $0.54-2.09)$. A novel measurement, Swiss ball four-point kneeling test was shown to be correlated with the change in BST $(r=0.633)$.
\end{abstract}

Keywords Asymmetry $\cdot$ Horse racing $\cdot$ Proprioception $\cdot$ Racing position $\cdot$ Swiss ball

$\begin{array}{ll}\text { Abbreviations } \\ \text { 95\% CI } & 95 \% \text { Confidence interval } \\ 95 \% \text { CL } & 95 \% \text { Confidence limit } \\ \text { BST } & \text { Biering-Sorensen test } \\ \text { CSTP } & \text { Core stabilization training program } \\ \text { cm } & \text { Centimeter } \\ d & \text { Cohens' } d \\ \text { kg } & \text { Kilogram } \\ \text { SBFPKT } & \text { Swiss ball four-point kneeling test } \\ \text { SD } & \text { Standard deviation } \\ \text { YBT } & \text { Y-Balance test }\end{array}$

Supplementary Information The online version contains supplementary material available at https://doi.org/10.1007/s1133 2-020-00729-0.

Nicholas H. K. Lam nicholas.lam@ed.ac.uk

1 Human Performance Science Research Group, Institute for Sport, PE and Health Sciences, The University of Edinburgh, Holyrood Road, Edinburgh EH88AQ, UK

2 Human Performance Laboratory, Technological and Higher Education Institute of Hong Kong (THEi), Chai Wan, Hong Kong

\section{Introduction}

Dynamic balance is the ability to adapt the movements based on the somatosensory system which provides visual and vestibular feedback to the brain to maintain the base of support [1]. The most common injuries among professional jockeys in British horse racing industry are acute head injury due to falling from the back of the horse, which is then followed by back and shoulder injuries which both are normally chronic injuries among jockeys [2]. An average of 9 million Australian dollar medical insurance claim per year was given to jockeys from 2002 to 2010 due to both acute and chronic injury [3]. This highlight the importance of dynamic balance and proprioception among horse riders.

Improving dynamic balance and back-extensor endurance may reduce the risk of fall during horse riding [4]. The lumbar segments were found to be tilting forward and backward synchronously during horse riding, which is likely to alter lower back and head position, while professional riders demonstrated minimal head and trunk movement compared to trainee riders [4]. Moreover, proprioception is vital, because human maintain posture equilibrium based on the vestibular and visual feedback 
provided by the somatosensory system $[1,5]$. Furthermore, Cullen et al. [6] also reported that horse riders need to adapt a racing position throughout the horse racing, which requires high neuromuscular control to produce maximal effort on the back of the horse. However, it is difficult to train the subconscious and unconscious movement through regular strength training due to the failure to generate unexpected turbulence [7].

Although traditional strength training could improve proprioception performance, previous research has shown that training in unstable condition could increase truck muscle activation and improve force exertion when experiencing unstable environments [8]. The implementation of balance pad and Swiss ball is a common method to imply unstable environment into training and has been shown to be able to improve core stability and proprioception $[9,10]$. The duration of 6-week training program focuses on core and dynamic posture presented positive improvement in the maximal reach of Y-balance test (YBT) and star excursion balance test $[11,12]$. YBT is a simplified version of Star Excursion Balance Test, and both the reach distance and asymmetry of YBT were shown to be related to risk of injury [13, 14]. Previous studies have indicated that core stability training program in unstable condition which involves Swiss ball and balance pad can improve core endurance and dynamic postural control [15-17]. Although 6-week training program with Swiss ball did not improve running economy and kinetics in runners [17], subjects became acutely aware of their body position after training [16]. Thus, we assume that performing exercise in unstable condition might reduce the risk of fall while maximizing the positive development of dynamic balance and upper trunk stabilization endurance for horse riders.

From these aforementioned studies, it is assumed that improving core stabilization endurance and dynamic balance could decrease the likelihood of acute and chronic injury. However, no studies have developed a horse-riding core training program to improve balance and core stabilization endurance to prevent the risk of fall. Therefore, the aim of this study was to investigate the effectiveness of a 6-week core stabilization training program (CSTP) on dynamic balance and back endurance performance of horse riders, and develop a measurement tool in assessing the dynamic postural stabilization endurance for horse riders. It hypothesized that the 6-week CSTP would improve dynamic balance and back extensors endurance of horse riders. In addition, regarding the body position of jockey riders during competitions, balance tests used in the previous studies were mostly in standing position rather than quadrupedal position. Thus, a more sport-specific balance test is required. The second hypothesis of the study is that Swiss ball four-point kneeling test (SBFPKT), a new assessment developed in this study, could be useful in examine horse riders' trunk stability, balance, and proprioception due to the similarity in posture.

\section{Methods}

\section{Participants}

12 male horse riders participated (age: $23.58 \pm 1.93$ years; height: $165.09 \pm 4.60 \mathrm{~cm}$; weight: $56.53 \pm 4.75 \mathrm{~kg}$; experience in horse racing: $1.75 \pm 0.34$ years). Inclusion criteria were aged between 18 and 30 years, $\geq 1$-year horse racing experience), injury free in the past 3 months. All subjects were informed about the testing procedures, and potential risks and benefits, and written informed consent and medical screening were completed before participation. The hypotheses of the study was disclosed at the end of the study. The research was approved by the Human Research Ethics Committee of The Technological and Higher Education Institute of Hong Kong and conducted in respect to the principles of the Declaration of Helsinki.

\section{Design}

A single-blind, case experimental design was applied. Subjects visited the Apprentice Jockey's School of the Hong Kong Jockey Club twice for 6 consecutive weeks excluding familiarization and post-test trial at similar time $( \pm 1 \mathrm{~h})$ with $\geq 48$ h between sessions. The CSTP lasted approximately 60 min per sessions for a total of 12 sessions. Anthropometric measurements were taken during familiarization trial and performed the entire testing protocol before pretest. Familiarization trial includes six practice trials in each direction in Y-Balance Test (YBT), three sets of $10 \mathrm{~s}$ for Biering-Sorensen test (BST) and SBFPKT to reduce the learning effect as suggested [18-20]. 10-min passive recovery was provided following the completion of the familiarization trial before conducting the pre-test. The testing order for YBT, BST, and SBPKT was randomized online (https:// www.graphpad.com/quickcalcs/randMenu/2.

\section{Methodology}

Subjects were instructed to avoid caffeine and alcohol consumption, and no vigorous physical activities at least $48 \mathrm{~h}$ prior experiment. All testing and training program were supervised and conducted by the same investigators to ensure consistency. Subjects performed a 10-min warm-up protocol before every session to minimize the risk of injury before trunk stabilization training with Swiss ball. The warm-up was modified from Sekendiz et al. [21] included 5 min walking followed by 2 sets of $15 \mathrm{~s}$ static stretching: kneeling trunk rotation, side lying hip adductor stretches, 
supine lying hamstring stretches, quadriceps and hip flexors stretches, and Child's pose. The cooldown was identical to warm-up protocol. Balance pad (Airex, Balance Pad Elite, $19.7^{\prime \prime} \times 16.1$ " $\times 2.4$ ", Germany) was used from the beginning of the training, while Swiss ball implemented (ChrisPower, Power Exercise Ball, Thailand) at week 3 and beyond.

\section{Y-balance test (YBT)}

YBT is a modified version of the Star Excursion Balance test in assessing dynamic balance and the asymmetry between limbs [19]. The YBT protocol used in this study was identical to Shaffer et al. [20] and Johnston et al. [18] as described. Participants stood in the center of the box with the foot aligning the anterior reach direction. Participants were instructed to use the non-standing leg to reach as far as possible without lifting the heel of the standing leg. Both hands were placed on the side of the hip throughout the test. The YBT was performed in the following sequence for both left and right legs: anterior, posteromedial, and posterolateral. The data were presented as left anterior (LA), left posteromedial (LPM), left posterolateral (LPL), right anterior (RA), right posteromedial (RPM), and right posterolateral (RPL). Measurement were conducted by the same investigator in pre- and post-testing sessions. YBT demonstrates good re-test reliability ( $\mathrm{ICC}=0.80-0.85$ ) in assessing dynamic balance [20]. This study reports the maximal reach distance and composite reach distance score of YBT $[18,20]$. The composite reach distance score of intra-rater and interrater reliability for YBT, is 0.91 and 0.99 , respectively [19].

The equation for the scoring system of YBT is in accordance with Shaffer et al. [20]:

Composite reach distance $(\%)=\frac{\text { Sum of the } 3 \text { reach directions }}{3 \times \text { limb length }} \times 100$.

\section{Biering-Sorensen test (BST)}

BST was used to examine the endurance capacity of the back-extensor muscle. The protocol used in this study was almost identical to previous research [22-24]. Subjects were asked to prone lying on the adjustable massage table (Coinfycare, CFTB02, China) with their body parts below anterior superior iliac spines strapped to the table at three locations: [22-24]. A chair was placed in front of the subjects for body adjustment and rest before beginning the test. The anterior superior iliac spines of the subjects were aligned with the edge of the table before tightening up the straps. A pillow was placed under both shins to reduce the space between legs and table. The straps were then firmly tightened up at ankle closest to the malleolus, at popliteal fossa of the knee, and at the level of the greater trochanter of the femur according to subject's level of comfort. The stationary arm of the goniometer was placed in line with the midline of the side of the subject's body and the moving arm was set at $170^{\circ}$. A white rigid tape with black marker on the tape was placed as close as possible to the goniometer on subjects' body to ensure that the body was positioned at $180^{\circ}$. The subjects were then told to lift the upper body with both arms across the chest and maintain the position as longer as possible. The time was calculated by stopwatch (Casio, HS-70W-1, Japan). The test was terminated when the marker dropped below $170^{\circ}$ or subject no longer continue because of exhaustion. No verbal encourage was given during the test. The BST demonstrates good reliability and interrater reliability ( $\mathrm{ICC}=0.85$ ) [22].

\section{Swiss ball four-point kneeling test (SBFPKT)}

As mentioned previously, the racing position for the final stage of the race is crucial [6]. Previous electromyography studies found that the implementation of Swiss ball reveals significantly higher muscles activation in lumbopelvic-hip complex including back-extensor muscles compared to exercise alone $[9,25]$. This shows that higher muscle activation is essential to overcome the unexpected turbulence during horse racing, which Swiss ball might be able to simulate the horse-riding situation. In addition to the similarity in posture, SBFPKT was developed to assess the horse riders' racing position performance which combined dynamic stability and trunk endurance. Two 55- or $65-\mathrm{cm}$ Swiss ball were pumped to the same pressure and the circumference was measured in the midline using measuring tape (Seca 206, Germany). 55-cm Swiss ball were used for those sub- jects with height range between 150 and $165 \mathrm{~cm}$, and $65-\mathrm{cm}$ Swiss ball were used for those subjects above $165 \mathrm{~cm}$ as recommended [9]. Subjects were told to kneel on one Swiss ball with the forearms placed on another Swiss ball. Arms and legs are expected to be flexed to approximately $90^{\circ}$ with forearms and lower limbs parallel to body, and clenched fists (Fig. 1). The time was calculated by stopwatch. Researcher started to record time when another research released their hands on the ball. The test was terminated when subjects fails to maintain approximately $90^{\circ}$ flexion for elbow and knee. No verbal encouragement was given during the test.

\section{Exercise intervention}

Subjects performed a supervised CSTP twice per week for 6 consecutive weeks. Each training session was approximately 


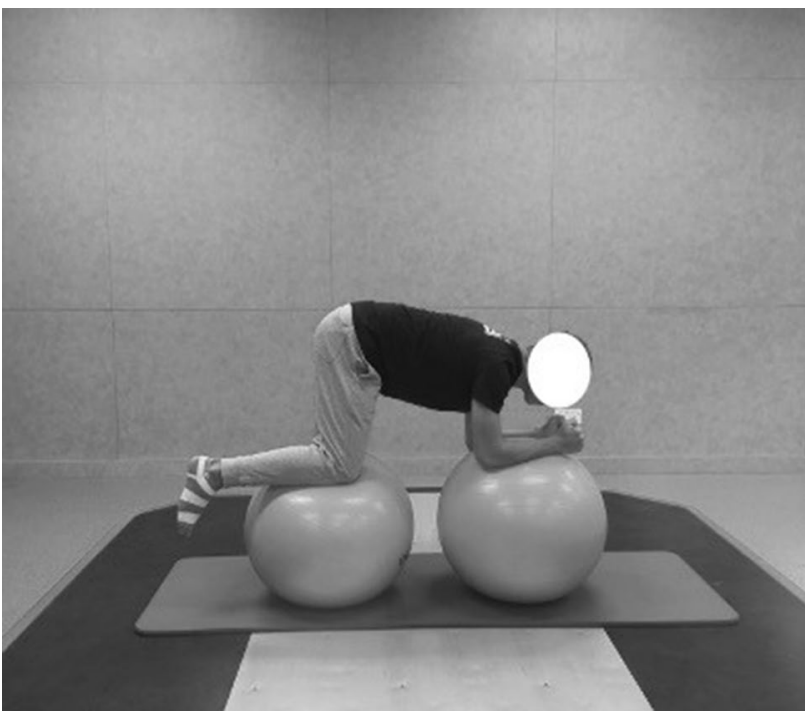

Fig. 1 Demonstration of the SBFPKT
$60 \mathrm{~min}$. There were two progressions with the implementation of Swiss ball and balance pad, which enhance muscle activation due to increased turbulence on unstable surface $[9,25]$. Consider the nature and activity level of the subjects, the progressions of the CSTP were started at level 2 of the 5-level approach for developing trunk stabilization training program as explained by Jeffreys [7]. The CSTP started with basic consciousness activation exercises, then eliminated visual feedback in dynamic balance task on unstable surfaces, and finished at moving the center of gravity without visual feedback to improve dynamic stability [21, 26]. Subjects progressed to the next level every 2 weeks to ensure appropriate muscle adaptation $[7,15,26]$. Therefore, exercises were remained similar, while the difficulties of the exercise increase synchronously to allow east transition for subjects. It also allows easy transition for subjects to complete new tasks. The protocol used in this is similar to previous research $[10,15]$. The details of the CSPT can be found in Table 1.
Table 1 Details of the 6-week CSTP: (a) the details of first 2 weeks of the core training program and (b) the details of weeks 3-6 of the core training program

\begin{tabular}{ll}
\hline Exercise & Sets/durations \\
\hline (a) Balance (perform with eyes opened) & \\
Single-leg shoulder bridge on BP & 3 sets $\times 30$ s each side (L/R) \\
Birddog on BP & 2 sets $\times 60$ s each side (L/R) \\
Kneeling on BP with trunk rotation & 2 sets $\times 30 \mathrm{~s}$ each side (L/R) \\
Kneeling on BP with straight arms & 1 set $\times 180 \mathrm{~s}$ \\
Single-leg kneeling on BP & 3 sets $\times 30 \mathrm{~s}$ each side (L/R) \\
3-min passive recovery & \\
Endurance & \\
Exercise & Sets/durations/rest period between sets \\
Shoulder bridge & 6 sets $\times 30 \mathrm{~s} / 15 \mathrm{~s}$ \\
Birddog & 6 sets $\times 30 \mathrm{~s} / 15 \mathrm{~s}$ \\
Dead bug & 6 sets $\times 30 \mathrm{~s} / 15 \mathrm{~s}$ \\
Curl-up & 6 sets $\times 30 \mathrm{~s} / 15 \mathrm{~s}$ \\
(b) Balance (perform with eyes closed) & \\
Single-leg shoulder bridge on BP & 3 sets $\times 30 \mathrm{~s}$ each side (L/R) \\
Birddog on BP* & 2 sets $\times 60 \mathrm{~s}$ each side (L/R) \\
Kneeling on BP with trunk rotation* & 2 sets $\times 30 \mathrm{~s}$ each side (L/R) \\
Kneeling on BP with straight arms* & 1 set $\times 180 \mathrm{~s}$ \\
Single-leg kneeling on BP & 3 sets $\times 30 \mathrm{~s} \mathrm{each} \mathrm{side} \mathrm{(L/R)}$ \\
3 -min passive recovery & \\
Endurance & \\
Exercise & Sets $/$ durations $/$ rest period between sets \\
Shoulder bridge with SB & 6 sets $\times 45 \mathrm{~s} / 15 \mathrm{~s}$ \\
Birddog with BP & 6 sets $\times 45 \mathrm{~s} / 15 \mathrm{~s}$ \\
Dead bug with SB & 6 sets $\times 45 \mathrm{~s} / 15 \mathrm{~s}$ \\
Curl-up with SB & 6 sets $\times 45 \mathrm{~s} / 15 \mathrm{~s}$ \\
\hline
\end{tabular}

$B P$ balance pad, $S B$ Swiss ball, SL: single leg

*Perform with head extension in week 5-6 


\section{Statistical analysis}

The null hypothesis significance testing fails to produce a practical meaning of the data due to the sample variability, and result in large variation of $p$ values [27]. Moreover, researchers should not make any practical assumption based on $p$ values to reduce overconfidence in small sample sizes as recommended [27-29]. In respect to the limitation of null hypothesis significance testing [28], alternative approach has been applied to circumvent the limitations of relying on $p$ value only. Data were presented as mean \pm standard deviation (SD) unless specified. Shapiro-Wilk test was used to assess the normality of the data. All variables were calculated using pairwise comparisons with the mean difference with $95 \%$ confidence interval (95\% CI) between pre- and post-condition. According to Lakens [30], mean SD demonstrates the optimal estimation of the population in a withinsubject study design which 95\% confidence limits (95\% CL) between trials should be reported along with effect sizes. The calculation method for 95\% confidence limits (95\%CL) was in line with Algina and Keselman [31]. Effect sizes were interpreted as the following: trivial $(d<0.2)$, small $(d \geq 0.2,<0.5)$, medium $(d \geq 0.5,<0.8)$, and large $(d \geq 0.8)$ [32]. The correlations between the change in SBFPKT performance from pre- to post-test and BST, anterior reach distance, posteromedial reach distance, posterolateral reach distance, composite score, and inter-limb difference were analyzed using Pearson's correlation coefficient. Correlations were classified as follows: small $(0.1-0.3)$, moderate $(0.3-0.5)$, large $(0.5-0.7)$, very large $(0.7-0.9)$, and extremely large (0.9-1.0) [33]. Statistical analyses were performed with SPSS 22.0 (IBM Corp., Armonk, NY) and Microsoft Excel ${ }^{\mathrm{TM}}$.

\section{Results}

Overall higher values were presented following the CSTP compared to pre-condition in YBT, BST, and SBFPKT. The maximal reach distance and inter-limb difference of YBT is presented in Table 2.
Large composite reach distance was observed in left leg compared to pre 6 weeks CSPT $(106.29 \pm 3.62$ vs $102.04 \pm 3.20$, mean difference 4.25 , 95\% CI 1.83-6.68, $d=1.25,95 \%$ CL $0.41-2.05$ ); and moderately differences were presented in right leg after 6 weeks CSPT $(106.471 \pm 4.35$ vs $102.81 \pm 8.32$, mean difference $3.66 \pm 5.72,95 \%$ CI $0.03-7.30, d=0.55,95 \%$ CL $0.00-1.08$ ). However, trivial to small differences was reported between left and right legs in pre (mean difference $0.77,95 \% \mathrm{CI}$ -3.50 to $5.04, d=0.12,95 \% \mathrm{CL}-0.49$ to 0.73 ) and post 6 weeks CSTP (mean difference $0.18,95 \%$ CI -3.13 to $3.48,95 \%$ CL -0.69 to 0.78 ). Paired sample $t$ test was also used to analyze the YBT difference between pre- and postCSPT. There were no significant differences in LA $(t=1.70$, $p=0.12), \mathrm{LPM}(t=1.55, p=0.15), \mathrm{LPL}(t=0.79, p=0.45)$ and RPM $(t=0.81, p=0.44)$. Wilcoxon signed-rank test shows no significant difference in RA $(z=1.37, p=0.169)$. The only significant difference between pre-test and posttest was found in YBTRPL, $t=-2.28, p=0.04, d=-0.66$.

The performance data of BST and SBFPKT are shown in Figs. 2 and 3. The difference in BST was large following the 6-week CSPT ( $d=1.33,95 \%$ CL $0.54-2.09$ ) and significant difference was presented $(t=-4.78, p=0.001)$. There was a moderate difference in SBFPKT after 6-week CSPT ( $d=0.79,95 \% \mathrm{CL}-0.05$ to 1.60$)$. Wilcoxon signed-rank test reports no significant difference between pre-test and post-test of SBFPKT $(z=-1.57, p=0.12)$.

The correlation between the change in SBFPKT performance and other test results is presented in Table 3. The improvement in post-SBFPKT is largely and positively correlated with BST $(r=0.633)$ and anterior reach distance of left leg $(r=0.558)$. Yet, the improvement in postSBFPKT has large and negative correlation with the posterolateral reach distance $(r=-0.532)$ and composite score $(r=-0.526)$ of right leg.

Table 2 Descriptive statistics for the maximal reach distance for each direction in Y-balance test after 6 weeks core stabilization training program

\begin{tabular}{lllccc}
\hline Performed limb & Direction & Before CSTP & After CSTP & Mean differences (95\% CI) & Cohens' $d(95 \%$ CL) \\
\hline Left & Anterior & $71.8 \pm 7.33$ & $75.8 \pm 5.70$ & $3.92 \pm 8.0(-1.17,9.0)$ & $0.60(-0.15,1.32)$ \\
& Posteromedial & $84.7 \pm 5.07$ & $88.6 \pm 5.88$ & $3.92 \pm 7.46(-0.83,8.66)$ & $0.71(-0.12,1.52)$ \\
& Posterolateral & $92.8 \pm 8.52$ & $95.3 \pm 5.31$ & $2.5 \pm 10.99(-4.48,9.48)$ & $0.35(-0.54,1.23)$ \\
Right & Anterior & $73.3 \pm 9.58$ & $76 \pm 5.86$ & $2.75 \pm 7.25(-1.86,7.36)$ & $0.35(-0.20,0.88)$ \\
& Posteromedial & $93.8 \pm 10.18$ & $96.1 \pm 5.09$ & $2.33 \pm 9.99(-4.01,8.68)$ & $0.29(-0.43,1.0)$ \\
& Posterolateral & $83.8 \pm 7.54$ & $87.7 \pm 5.35$ & $3.83 \pm 5.81(0.14,7.53)$ & $0.59(0.02,1.13)$ \\
\hline
\end{tabular}




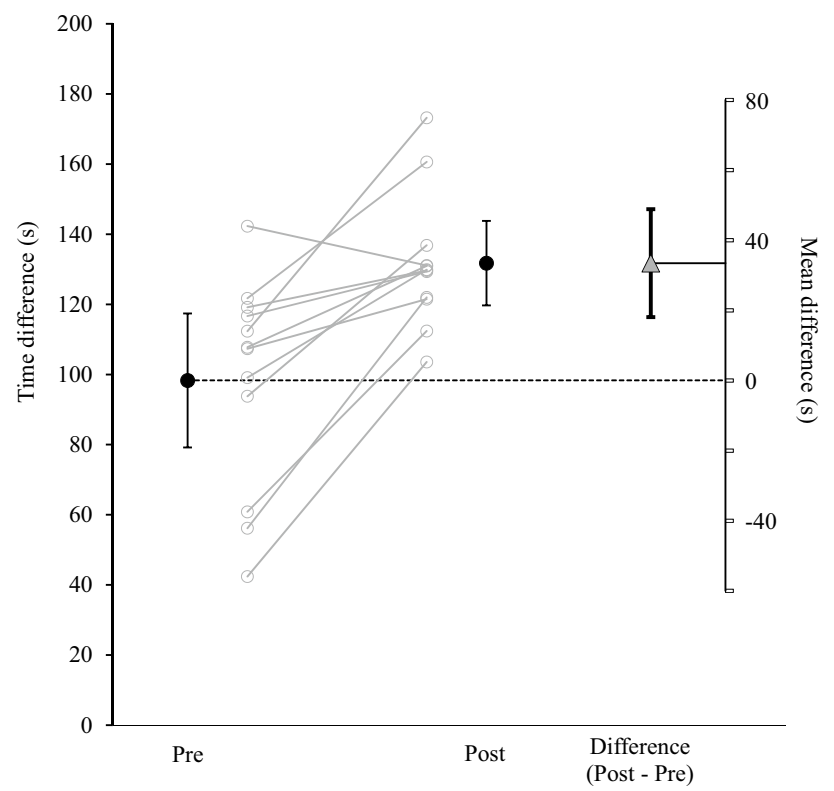

Fig. 2 Performance of the BST before and after 6-week CSTP. Black circles with error bars are presented as mean value with $95 \% \mathrm{CI}$; gray lines represent individual participant values; gray triangle represents the mean difference between conditions with 95\% CI

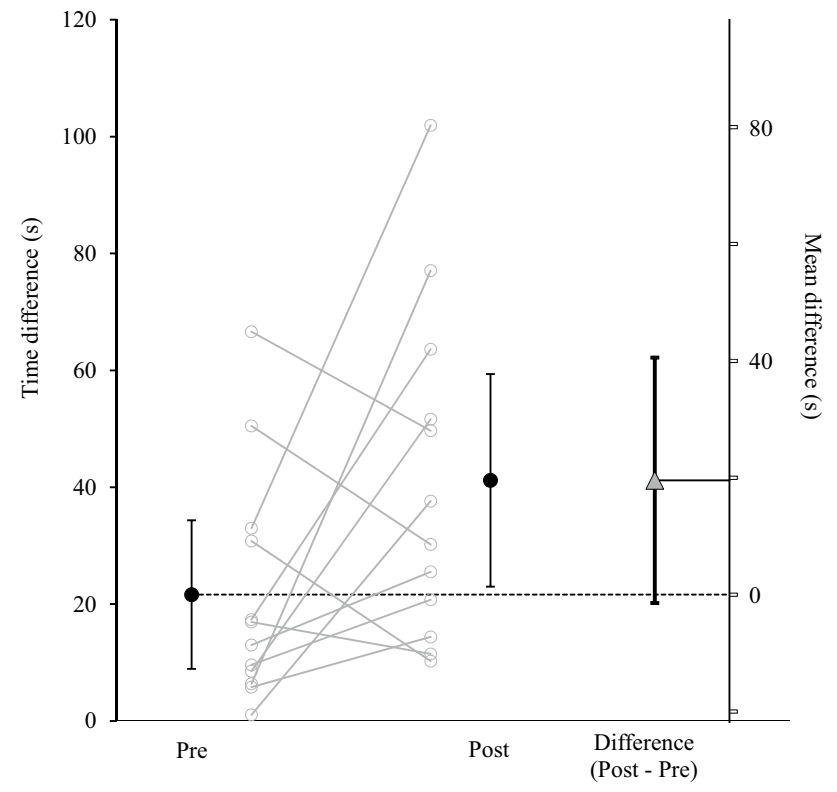

Fig. 3 Performance of the SBFPKT before and after 6-week CSTP. Black circles with error bars are presented as mean value with $95 \%$ $\mathrm{CI}$; gray lines represent individual participant values; gray triangle represents the mean difference between conditions with 95\% CI

\section{Discussion}

This study investigated the efficacy of the 6-week trunk stabilization training program on dynamic balance and

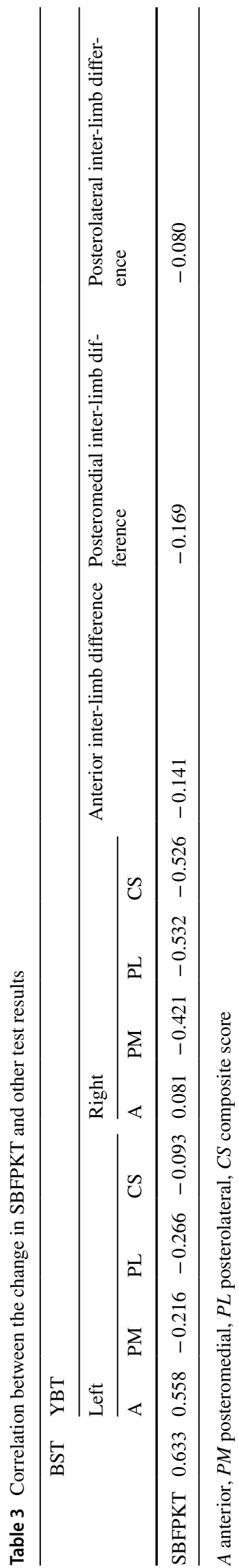


back-extensor endurance of horse riders. The result obtained in this study was in accordance with the hypotheses, 6-week CSTP largely improved back-extensor endurance performance in BST and the composite reach distance of left leg in YBT. The inter-limb difference of anterior reach and posteromedial reach was decreased moderately. Whilst maximal reach distance and composite reach distance of right leg were small to moderately greater after CSTP. We also observed a moderate improvement in SBFPKT, which has large and positive correlation with the improvement in BST.

\section{Dynamic balance performance}

We observed small and moderate increases in maximal reach distance following the 6-week CSTP, right and left leg, respectively. Large improvement is also reported in YBT after 6-week CSTP with two progressions includes eliminating visual feedback and switching center of gravity. Our findings are consistent with the literature $[9,34$, 35]. The novel finding of our study is that changing the training surface and reducing visual feedback in trunk stabilization exercises could be effective on improving dynamic balance, which aligned with previous studies [34-36]. The improvement in dynamic balance after 6-week CSTP could be explained in the following reasons. First, the horse racing experience of the subjects was considered as beginner ( $\geq 1$-year horse racing experience) which is expected to have a slightly poor balance performance compared to well-trained horse riders. Thus, the potential of improvement could be greater compared to experienced horse riders. Bagherian et al. [34] highlighted that poor-quality mover in dynamic balance task may elicit greater improvement following trunk stability training, because improved trunk stability could enhance the neuromuscular control of the lower limb [37]. Second, biofeedback has been shown to be related to postural control especially when the exercise requires high motion variability, which presents higher trunk translation if visual feedback is eliminated [38]. Therefore, it is plausible to assume that dynamic balance could be improved through adjusting visual feedback during exercise. Interestingly, increased YBT composite score and maximal reach distance together with a reduced mean difference between limb were observed after 6 weeks CSTP, which suggests that trunk stabilization training with the progression of implementing unstable training surface may reduce the functional asymmetry in the lower limbs. As asymmetry of dynamic balance performance in lower extremities was suggested to be a risk of injury [14], we can assume that CSTP that involves the use of unstable surface can potentially decrease the injury risk.

\section{Effectiveness of Swiss ball and balance pad}

We also observed that almost all subjects presented moderate-to- large changes in SBFPKT and BST which is in line with our hypotheses. The potential benefit of Swiss ball and balance pad may be due to the increased muscle activation on unstable training surface $[9,25]$, which explain the positive changes in SBFPKT. Indeed, it has already been demonstrated that exercises involved switching the base of support and unexpected turbulence would improve muscle maximal voluntary contraction [36]. The involvement of Swiss ball and balance pad in our 6-week CSTP was likely to produce positive effect not only in dynamic balance but also trunk endurance including back-extensor endurance. This suggests that the combination of balance and trunk stability exercise would increase the neuromuscular control of the lower extremities as mentioned [37]. These data indicated that 6-week CSTP had a positive impact on subjects' dynamic balance and back-extensor endurance. Consequently, training programs with the progression of eliminating visual feedbacks and changing training surface can promote the development of proprioception, and possibly reduce the risk of fall. We clearly demonstrated that 6-week CSTP can potentially increase neuromuscular control of the lower limb and trunk stability in a short period of time. To ensure that CSTP combine well with regular training program, it may be advantageous to know whether isolating balance or trunk endurance training would elicit the same effects.

Despite all test results improved throughout the 6-week CSTP, only the improvement of BST and anterior reach were positively correlated with the change in SBFPKT. Previous research has shown that plank with either arms or legs on unstable surface can elicit higher EMG amplitude in erector spinae compared to stable condition [39]. Due to the fact that trunk extensor works as one of the trunk stabilizers in prone posture on unstable surface, we can assume that SBFPKT is a good assessment of trunk extensor in unstable condition. Although the similarity between racing position and SBFPKT is theoretically acceptable, future studies can consider implementing motion analysis and EMG measurement for comparison to improve the validity of the SBFPKT. For the correlation between changes in SBFPKT and YBT, the result is contrasting among limbs and directions. Such inconsistency of correlation can be attributed to the following reasons. First, the posture is different between SBFPKT and YBT. The SBFPKT was executed in prone position and the YBT was in standing position. Previous studies have suggested that trunk strength and stability examine in prone position (e.g., plank, BST) were not correlated with star excursion balance test $[40,41]$. Also, the YBT may be affected by other factors including lower extremities strength and range of motion $[42,43]$. Thus, we suggest that YBT should not be solely used to assess jockey riders' trunk 
stability. The other reason is that the sample size is small and the participants' experience as jockey rider is insufficient, which may lead to inconsistent level of physical performance, causing the correlation coefficient to be limited. Future work should consider recruiting larger sample size of jockey riders with both novice and professional level riders.

\section{Limitations}

This study had several limitations. As mentioned in discussion, the sample size was limited by the population of jockey riders in Hong Kong. Thus, the result may not be able to represent the actual population. Moreover, the participants were all male novice rider, and strength and conditioning coaches should imply the result of the current study cautiously on female and experienced riders. The authors also failed to measure the range of motion of each participant, and thus, it would be inappropriate to assume that the change in YBT performance is solely attributed to the improvement in trunk stability. Finally, the current study did not recruit control group or compare it CSTP with other training methods. Future studies should consider doing so to ensure that CSTP is more efficient than other methods.

\section{Practical applications}

The 6-week CSTP with the progression of eliminating visual feedback and switching center of gravity appears to be useful in improving horse riders dynamic balance, back-extensor endurance performance, and functional asymmetry in the lower limb. Coaches should consider implementing balance and trunk stabilization-based training into regular training to reduce the injury incidence in horse riding, particularly 6 weeks before competition. A novel method of measuring dynamic balance incorporating with the standardization of Swiss ball has been developed and named SBFPKT. The present finding suggests that the reliability and validity of SBFPKT should be investigated to predict the racing performance of horse riders.

\section{Conclusions}

To conclude, the 6-week CSTP focused on dynamic balance and trunk endurance improves back-extensor endurance and both bilateral and unilateral dynamic balance of lower limbs. To our knowledge, this is also the first study using Swiss ball as a measurement to assess racing position of the horse riders. Authors believe that the SBFPKT could be a meaningful method in evaluating riders' trunk stability in prone and unstable position, which is similar to the racing position of jockey riders.
Funding No funding was received for this study.

\section{Compliance with ethical standards}

Conflict of interest The authors report no conflicts of interest related to the work.

Ethical approval The research was approved by the Human Research Ethics Committee of The Technological and Higher Education Institute of Hong Kong and conducted in respect to the principles of the Declaration of Helsinki.

Informed consent All subjects were informed about the testing procedures, and potential risks and benefits, and written informed consent and medical screening were completed before participation.

Open Access This article is licensed under a Creative Commons Attribution 4.0 International License, which permits use, sharing, adaptation, distribution and reproduction in any medium or format, as long as you give appropriate credit to the original author(s) and the source, provide a link to the Creative Commons licence, and indicate if changes were made. The images or other third party material in this article are included in the article's Creative Commons licence, unless indicated otherwise in a credit line to the material. If material is not included in the article's Creative Commons licence and your intended use is not permitted by statutory regulation or exceeds the permitted use, you will need to obtain permission directly from the copyright holder. To view a copy of this licence, visit http://creativecommons.org/licenses/by/4.0/.

\section{References}

1. Ogard KW (2011) Proprioception in sports medicine and athletic conditioning. Strength Cond J 33(3):111-118. https://doi. org/10.1519/SSC.0b013e31821bf3ae

2. Balendra G, Turner M, McCrory P (2008) Career-ending injuries to professional jockeys in British horse racing (1991-2005). Br J Sports Med 42(1):22. https://doi.org/10.1136/bjsm.2007.038950

3. Beverley AC, Peta LH, Petr O, Lei S, Andrew JP (2015) Workplace injuries in thoroughbred racing: an analysis of insurance payments and injuries amongst Jockeys in Australia from 2002 to 2010. Animals 5(3):897-909. https://doi.org/10.3390/ani5030390

4. Olivier A, Faugloire E, Lejeune L, Biau S, Isableu B (2017) Head stability and head-trunk coordination in horseback riders: the contribution of visual information according to expertise. Front Hum Neurosci. https://doi.org/10.3389/fnhum.2017.00011

5. Lee T, Kim YH, Sung PS (2011) A comparison of pain level and entropy changes following core stability exercise intervention. Med Sci Monit 17(7):CR362. https://doi.org/10.12659/ MSM.881846

6. Cullen DS, O'loughlin DG, McGoldrick DA, Smyth DB, May DG, Warrington DG (2015) Physiological demands of flat horse racing Jockeys. J Strength Cond Res 29(11):3060-3066. https:// doi.org/10.1519/JSC.0000000000000977

7. Jeffreys I (2002) Developing a progressive core stability program. Strength Cond J 24(5):65-66. https://doi.org/10.1519/15334295(2002)024\%3c0065:DAPCSP\%3e2.0.CO;2

8. Behm D, Colado JC (2012) The effectiveness of resistance training using unstable surfaces and devices for rehabilitation. Int J Sports Phys Ther 7(2):226 
9. Escamilla RF, Lewis C, Bell D, Bramblet G, Daffron J, Lambert S, Pecson A, Imamura R, Paulos L, Andrews JR (2010) Core muscle activation during Swiss ball and traditional abdominal exercises. J Orthop Sports Phys Ther 40(5):265-276

10. Nowotny A, Calderon M, Albuquerque de Souza P, Léonard G, Oliveira Alves B, Da Silva R (2018) Lumbar stabilisation exercises versus back endurance-resistance exercise training in athletes with chronic low back pain: protocol of a randomised controlled trial. BMJ Open Sport Exerc Med. https://doi.org/10.1136/bmjse m-2018-000452

11. Dhvani NS, Annamma V (2014) Effect of core stability training on dynamic balance in healthy young adults - a randomized controlled triaL. Int J Physiother 1(4):187-194. https://doi. org/10.15621/ijphy/2014/v1i4/54563

12. Kahle N, Gribble P (2009) Core stability training in dynamic balance testing among young, healthy adults. Athl Train Sports Health Care 1(2):65-73. https://doi.org/10.3928/19425864-20090 301-03

13. Gonell AC, Romero JAP, Soler LM (2015) Relationship between the y balance test scores and soft tissue injury incidence in a soccer team. Int J Sports Phys Ther 10(7):955

14. Smith AC, Chimera JN, Warren JM (2015) Association of Y balance test reach asymmetry and injury in division I athletes. Med Sci Sports Exerc 47(1):136-141. https://doi.org/10.1249/ MSS.0000000000000380

15. Sandrey M, Mitzel J (2013) Improvement in dynamic balance and core endurance after a 6-week core-stability-training program in high school track and field athletes. J Sport Rehabil 22(4):264271. https://doi.org/10.1123/jsr.22.4.264

16. Sato K, Mokha M (2009) Does core strength training influence running kinetics, lower-extremity stability, and 5000-m performance in runners? J Strength Cond Res 23(1):133-140. https:// doi.org/10.1519/JSC.0b013e31818eb0c5

17. Stanton RR, Reaburn RP, Humphries RB (2004) The effect of short-term Swiss ball training on core stability and running economy. J Strength Cond Res 18(3):522-528. https://doi. org/10.1519/1533-4287(2004)182.0.CO;2

18. Johnston W, Dolan K, Reid N, Coughlan GF, Caulfield B (2018) Investigating the effects of maximal anaerobic fatigue on dynamic postural control using the Y-balance test. J Sci Med Sport 21(1):103-108. https://doi.org/10.1016/j.jsams.2017.06.007

19. Plisky PJ, Gorman PP, Butler RJ, Kiesel KB, Underwood FB, Elkins B (2009) The reliability of an instrumented device for measuring components of the star excursion balance test. $\mathrm{N}$ Am J Sports Phys Ther NAJSPT 4(2):92

20. Shaffer SW, Teyhen DS, Lorenson CL, Warren RL, Koreerat CM, Straseske CA, Childs JD (2013) Y-balance test: a reliability study involving multiple raters. Mil Med 178(11):1264. https:// doi.org/10.7205/MILMED-D-13-00222

21. Sekendiz B, Cuğ M, Korkusuz F (2010) Effects of Swiss-ball core strength training on strength, endurance, flexibility, and balance in sedentary women. J Strength Cond Res 24(11):3032-3040. https ://doi.org/10.1519/JSC.0b013e3181d82e70

22. Latimer GJ, Maher GC, Refshauge GK, Colaco GI (1999) The reliability and validity of the Biering-Sorensen test in asymptomatic subjects and subjects reporting current or previous nonspecific low back pain. Spine 24(20):2085-2085. https://doi. org/10.1097/00007632-199910150-00004

23. Pitcher M, Behm D, Mackinnon S (2007) Neuromuscular fatigue during a modified Biering-Sørensen test in subjects with and without low back pain. J Sports Sci Med 6(4):549-559

24. Tekin Y, Ortancil O, Ankarali H, Basaran A, Sarikaya S, Ozdolap S (2009) Biering-Sorensen test scores in coal miners. Jt Bone Spine 76(3):281-285. https://doi.org/10.1016/j.jbspi n.2008.08.008
25. Marshall PW, Murphy BA (2008) Muscle activation changes after exercise rehabilitation for chronic low back pain. Arch Phys Med Rehabil 89(7):1305-1313. https://doi.org/10.1016/j. apmr.2007.11.051

26. Fort-Vanmeerhaeghe SA, Romero-Rodriguez DD, Lloyd DR, Kushner DA, Myer DG (2016) Integrative neuromuscular training in youth athletes. Part II: strategies to prevent injuries and improve performance. Strength Cond J 38(4):9-27. https://doi. org/10.1519/SSC.0000000000000234

27. Wasserstein R, Schirm A, Lazar N (2019) Moving to a World Beyond "p < 0.05." Am Stat 73(s1):1-19. https://doi. org/10.1080/00031305.2019.1583913

28. Calin-Jageman R, Cumming G (2019) The new statistics for better science: ask how much, how uncertain, and what else is known. Am Stat 73(1):271-280. https://doi.org/10.1080/00031 305.2018.1518266

29. Wasserstein R, Lazar N (2016) The ASA's statement on p-values: context, process, and purpose. Am Stat 70(2):129-133. https://doi. org/10.1080/00031305.2016.1154108

30. Lakens D (2013) Calculating and reporting effect sizes to facilitate cumulative science: a practical primer for t-tests and ANOVAs. Front Psychol. https://doi.org/10.3389/fpsyg.2013.00863

31. Algina J, Keselman J (2003) Approximate confidence intervals for effect sizes. Educ Psychol Meas 63(4):537-553. https://doi. org/10.1177/0013164403256358

32. Cohen J (1992) A power primer. Psychol Bull 112(1):155-159. https://doi.org/10.1037/0033-2909.112.1.155

33. Hopkins GW, Marshall WS, Batterham MA, Hanin MJ (2009) Progressive statistics for studies in sports medicine and exercise science. Med Sci Sports Exerc 41(1):3-12. https://doi. org/10.1249/MSS.0b013e31818cb278

34. Bagherian S, Ghasempoor K, Rahnama N, Wikstrom E (2019) The effect of core stability training on functional movement patterns in College athletes. J Sport Rehabil 28(5):444-449. https:// doi.org/10.1123/jsr.2017-0107

35. Granacher U, Schellbach J, Klein K, Prieske O, Baeyens J-P, Muehlbauer T (2014) Effects of core strength training using stable versus unstable surfaces on physical fitness in adolescents: a randomized controlled trial. BMC Sports Sci Med Rehabil 6(1):40. https://doi.org/10.1186/2052-1847-6-40

36. Mayer JM, Quillen WS, Verna JL, Chen R, Lunseth P, Dagenais S (2015) Impact of a supervised worksite exercise program on back and core muscular endurance in firefighters. Am J Health Promot 29(3):165-172. https://doi.org/10.4278/ajhp.130228-QUAN-89

37. Sasaki S, Tsuda E, Yamamoto Y, Maeda S, Kimura Y, Fujita Y, Ishibashi Y (2019) Core-muscle training and neuromuscular control of the lower limb and trunk. J Athl Train 54(9):959. https:// doi.org/10.4085/1062-6050-113-17

38. Hamacher D, Hamacher D, Schega L (2015) Does visual augmented feedback reduce local dynamic stability while walking? Gait Posture 42(4):415-418. https://doi.org/10.1016/j.gaitp ost.2015.07.007

39. Snarr LR, Esco RM (2014) Electromyographical comparison of plank variations performed with and without instability devices. J Strength Cond Res 28(11):3298-3305. https://doi.org/10.1519/ JSC.0000000000000521

40. Gordon AT, Ambegaonkar JP, Caswell SV (2013) Relationships between core strength, hip external rotator muscle strength, and star excursion balance test performance in female lacrosse players. Int J Sports Phys Ther 8(2):97

41. Ozmen T (2016) Relationship between core stability, dynamic balance and jumping performance in soccer players. Turk J Sport Exerc 18(1):110-113

42. Lee D-K, Kim G-M, Ha S-M, Oh J-S (2014) Correlation of the Y-balance test with lower-limb strength of adult women. J Phys Ther Sci 26(5):641. https://doi.org/10.1589/jpts.26.641 
43. López-Valenciano A, Ayala F, De Ste CM, Barbado D, VeraGarcia F (2019) Different neuromuscular parameters influence dynamic balance in male and female football players. Knee Surg Sports Traumatol Arthrosc 27(3):962-970. https://doi. org/10.1007/s00167-018-5088-y
Publisher's Note Springer Nature remains neutral with regard to jurisdictional claims in published maps and institutional affiliations. 